\title{
Design Principles for HFEv- based Multivariate Signature Schemes
}

\author{
No Author Given \\ No Institute Given
}

\begin{abstract}
The Hidden Field Equations (HFE) Cryptosystem as proposed by Patarin is one of the best known and most studied multivariate schemes. While the security of the basic scheme appeared to be very weak, the HFEv- variant seems to be a good candidate for digital signature schemes on the basis of multivariate polynomials. However, the currently existing scheme of this type, the QUARTZ signature scheme, is hardly used in practice because of its poor efficiency. In this paper we analyze recent results from Ding and Yang about the degree of regularity of HFEv- systems and derive from them design principles for signature schemes of the HFEv- type. Based on these results we propose the new HFEv- based signature scheme Gui, which is more than 100 times faster than QUARTZ and therefore comparable with classical signature schemes such as RSA and ECDSA.
\end{abstract}

Keywords: Multivariate Cryptography, Digital Signatures, HFEv-, Design Principles, Security, Performance

\section{Introduction}

Cryptographic techniques are an essential tool to guarantee the security of communication in modern society. Today, the security of nearly all of the cryptographic schemes used in practice is based on number theoretic problems such as factoring large integers and solving discrete logarithms. The best known schemes in this area are RSA [25], DSA [16] and ECC. However, schemes like these will become insecure as soon as large enough quantum computers arrive. The reason for this is Shor's algorithm [26], which solves number theoretic problems like integer factorization and discrete logarithms in polynomial time on a quantum computer. Therefore, one needs alternatives to those classical public key schemes, based on hard mathematical problems not affected by quantum computer attacks.

Besides lattice, code and hash based cryptosystems, multivariate cryptography is one of the main candidates for this [1]. Multivariate schemes are in general very fast and require only modest computational resources, which makes them attractive for the use on low cost devices like smart cards and RFID chips $[4,5]$. Additionally, at least in the area of digital signatures, there exists a large number 
of practical multivariate schemes $[10,17]$.

In 2001, Patarin and Courtois proposed a multivariate signature scheme called QUARTZ [21], which is based on the concept of HFEv-. While QUARTZ produces very short signatures (128 bit), the signature generation process is very slow (at the time about 11 seconds per signature [5]). The main reason for this is the use of a high degree HFE polynomial (for QUARTZ this degree is given by $D=129$ ), which makes the inversion of the central map very costly.

At the time of the design of the QUARTZ scheme, very little was known about the complexity of algebraic attacks against the HFE family of systems, in particular, the HFEv- schemes. Therefore, the authors of QUARTZ could not base their parameter choice on theoretical foundations. Recently, there has been a fundamental breakthrough in terms of understanding the behavior of algebraic attacks on the HFE family of systems $[8,9,11]$, which gives an upper bound of the degree of regularity of Gröbner basis attacks against those schemes.

In this paper, we review and analyze the results of Ding and Yang and derive from these results design criteria for HFEv- based signature schemes. In particular we show that we can, by increasing the numbers $a$ of Minus equations and $v$ of Vinegar variables, achieve adequate security even for low degree HFE polynomials and that the upper bound on the degree of regularity given by Ding and Yang is reasonably tight. Based on our analysis, we propose the new HFEv- based signature scheme Gui ${ }^{1}$, which uses an HFE polynomial of very small degree, namely $D \in\{5,9,17\}$. This enables us to speed up the signature generation process of QUARTZ by a factor of more than 100, without weakening the security of the scheme. By doing so, we create a highly practical multivariate signature scheme, whose performance is comparable to that of classical signature schemes such as RSA and ECDSA.

The rest of this paper is organized as follows. In Section 2 we give an introduction into the area of multivariate cryptography and in particular Big-Field signature schemes. Section 3 introduces the HFEv- signature scheme and the changes made to this scheme by Patarin and Courtois when defining QUARTZ. Furthermore, in this section, we discuss the performance and the security of HFEv- based signature schemes. In Section 4 we analyze the results of Ding and Yang on the behaviour of direct attacks on HFEv- schemes by performing a large number of experiments and present the design criteria we derive from that. Based on these results, we propose in Section 5 our new multivariate signature scheme Gui. Section 6 gives details on the implementation of the scheme and compares the efficiency of Gui with that of some standard signature schemes. Finally, Section 7 concludes the paper.

\footnotetext{
${ }^{1}$ We call our new scheme Gui, after tripod earthenware pottery dating back to the 4000-year-old Longshan culture [28].
} 


\section{Multivariate Cryptography}

The basic objects of multivariate cryptography are systems of multivariate quadratic polynomials. (see equation (1)).

$$
\begin{gathered}
p^{(1)}\left(x_{1}, \ldots, x_{n}\right)=\sum_{i=1}^{n} \sum_{j=i}^{n} p_{i j}^{(1)} \cdot x_{i} x_{j}+\sum_{i=1}^{n} p_{i}^{(1)} \cdot x_{i}+p_{0}^{(1)} \\
p^{(2)}\left(x_{1}, \ldots, x_{n}\right)=\sum_{i=1}^{n} \sum_{j=i}^{n} p_{i j}^{(2)} \cdot x_{i} x_{j}+\sum_{i=1}^{n} p_{i}^{(2)} \cdot x_{i}+p_{0}^{(2)} \\
\vdots \\
p^{(m)}\left(x_{1}, \ldots, x_{n}\right)=\sum_{i=1}^{n} \sum_{j=i}^{n} p_{i j}^{(m)} \cdot x_{i} x_{j}+\sum_{i=1}^{n} p_{i}^{(m)} \cdot x_{i}+p_{0}^{(m)}
\end{gathered}
$$

The security of multivariate schemes is based on the

MQ Problem: Given $m$ multivariate quadratic polynomials $p^{(1)}(\mathbf{x}), \ldots, p^{(m)}(\mathbf{x})$ in $n$ variables $x_{1}, \ldots, x_{n}$, find a vector $\overline{\mathbf{x}}=\left(\bar{x}_{1}, \ldots, \bar{x}_{n}\right)$ such that $p^{(1)}(\overline{\mathbf{x}})=\ldots=$ $p^{(m)}(\overline{\mathbf{x}})=0$.

The MQ problem (for $m \approx n$ ) is proven to be NP-hard even for quadratic polynomials over the field $\mathrm{GF}(2)$ [14].

To build a public key cryptosystem based on the MQ problem, one starts with an easily invertible quadratic map $\mathcal{F}: \mathbb{F}^{n} \rightarrow \mathbb{F}^{m}$ (central map). To hide the structure of $\mathcal{F}$ in the public key, one composes it with two invertible affine (or linear) maps $\mathcal{S}: \mathbb{F}^{m} \rightarrow \mathbb{F}^{m}$ and $\mathcal{T}: \mathbb{F}^{n} \rightarrow \mathbb{F}^{n}$. The public key is therefore given by $\mathcal{P}=\mathcal{S} \circ \mathcal{F} \circ \mathcal{T}$. The private key consists of $\mathcal{S}, \mathcal{F}$ and $\mathcal{T}$ and therefore allows to invert the public key.

Note: Due to the above construction, the security of multivariate schemes is not only based on the MQ-Problem but also on the EIP-Problem ("Extended Isomorphism of Polynomials") of finding the composition of $\mathcal{P}$.

In this paper we concentrate on multivariate signature schemes of the BigField family. For this type of multivariate schemes, the map $\mathcal{F}$ is a specially chosen easily invertible map over a degree $n$ extension field $\mathbb{E}$ of $\mathbb{F}$. One uses an isomorphism $\Phi: \mathbb{F}^{n} \rightarrow \mathbb{E}$ to transform $\mathcal{F}$ into a quadratic map

$$
\overline{\mathcal{F}}=\Phi^{-1} \circ \mathcal{F} \circ \Phi
$$

from $\mathbb{F}^{n}$ to itself. The public key of the scheme is therefore given by

$$
\mathcal{P}=\mathcal{S} \circ \overline{\mathcal{F}} \circ \mathcal{T}=\mathcal{S} \circ \Phi^{-1} \circ \mathcal{F} \circ \Phi \circ \mathcal{T}: \mathbb{F}^{n} \rightarrow \mathbb{F}^{n} .
$$

The standard signature generation and verification process of a multivariate BigField scheme works as shown in Figure 1. 


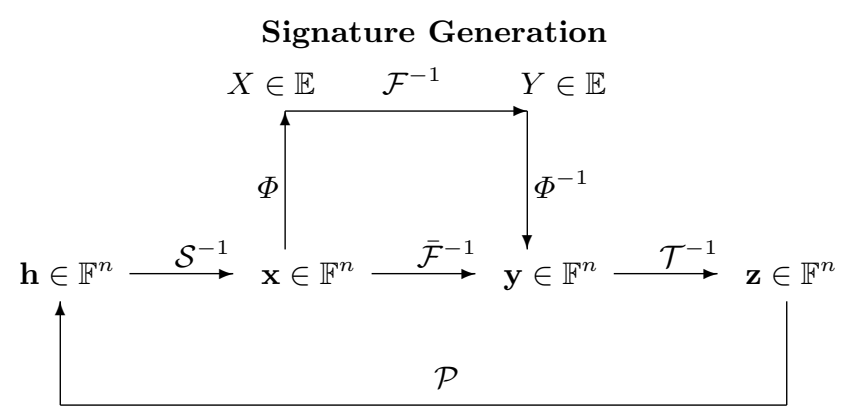

Signature Verification

Fig. 1. General workflow of multivariate BigField signature schemes

Signature generation: To generate a signature for a message $\mathbf{h} \in \mathbb{F}^{n}$, one computes recursively $\mathbf{x}=\mathcal{S}^{-1}(\mathbf{h}) \in \mathbb{F}^{n}, X=\Phi(\mathbf{x}) \in \mathbb{E}, Y=\mathcal{F}^{-1}(X) \in \mathbb{E}$, $\mathbf{y}=\Phi^{-1}(Y) \in \mathbb{F}^{n}$ and $\mathbf{z}=\mathcal{T}^{-1}(\mathbf{y})$. The signature of the message $\mathbf{h}$ is $\mathbf{z} \in \mathbb{F}^{n}$.

Verification: To check the authenticity of a signature $\mathbf{z} \in \mathbb{F}^{n}$, one simply computes $\mathbf{h}^{\prime}=\mathcal{P}(\mathbf{z}) \in \mathbb{F}^{n}$. If $\mathbf{h}^{\prime}=\mathbf{h}$ holds, the signature is accepted, otherwise rejected.

A good overview on existing multivariate schemes can be found in [7].

Two widely used variations of multivariate BigField signature schemes are the Minus variation and the use of additional (Vinegar) variables.

Minus-Variation: The idea of this variation is to remove a small number of equations from the public key. The Minus-Variation was first used in schemes like SFLASH [22] to prevent Patarins Linearization Equations attack [23] against the Matsumoto-Imai cryptosystem [20].

Vinegar-Variation: The idea of this variation is to parametrize the central map $\mathcal{F}$ by adding (a small set of) additional (Vinegar) variables. In the context of multivariate BigField signature schemes, the Vinegar variation can be used to increase the security of the scheme against direct and rank attacks. 


\section{The HFEv- Signature Scheme}

In this section we introduce the HFEv- signature scheme, which is the basis of QUARTZ and our new signature scheme Gui (see Section 5).

Let $\mathbb{F}=\mathbb{F}_{q}$ be a finite field with $q$ elements and $\mathbb{E}$ be a degree $n$ extension field of $\mathbb{F}$. Furthermore, we choose integers $D, a$ and $v$. Let $\Phi$ be the canonical isomorphism between $\mathbb{F}^{n}$ and $\mathbb{E}$, i.e.

$$
\Phi\left(x_{1}, \ldots, x_{n}\right)=\sum_{i=1}^{n} x_{i} \cdot X^{i-1} .
$$

The central map $\mathcal{F}$ of the HFEv- scheme is a map from $\mathbb{E} \times \mathbb{F}^{v}$ to $\mathbb{E}$ of the form

$$
\begin{aligned}
\mathcal{F}(X) & =\sum_{0 \leq i \leq j}^{q^{i}+q^{j} \leq D} \alpha_{i j} \cdot X^{q^{i}+q^{j}} \\
& +\sum_{i=0}^{q^{i} \leq D} \beta_{i}\left(v_{1}, \ldots, v_{v}\right) \cdot X^{q^{i}} \\
& +\gamma\left(v_{1}, \ldots, v_{v}\right)
\end{aligned}
$$

with $\alpha_{i j} \in \mathbb{E}, \beta_{i}: \mathbb{F}^{v} \rightarrow \mathbb{E}$ being linear and $\gamma: \mathbb{F}^{v} \rightarrow \mathbb{E}$ being a quadratic function.

Due to the special form of $\mathcal{F}$, the map $\overline{\mathcal{F}}=\Phi^{-1} \circ \mathcal{F} \circ \Phi$ is a quadratic polynomial map from $\mathbb{F}^{n+v}$ to $\mathbb{F}^{n}$. To hide the structure of $\overline{\mathcal{F}}$ in the public key, one combines it with two affine (or linear) maps $\mathcal{S}: \mathbb{F}^{n} \rightarrow \mathbb{F}^{n-a}$ and $\mathcal{T}: \mathbb{F}^{n+v} \rightarrow \mathbb{F}^{n+v}$ of maximal rank.

The public key of the scheme is the composed map $\mathcal{P}=\mathcal{S} \circ \overline{\mathcal{F}} \circ \mathcal{T}: \mathbb{F}^{n+v} \rightarrow \mathbb{F}^{n-a}$, the private key consists of $\mathcal{S}, \mathcal{F}$ and $\mathcal{T}$.

Signature generation: To generate a signature for a message $\mathbf{h} \in \mathbb{F}^{n-a}$, the signer performs the following three steps.

1. Compute a preimage $\mathbf{x} \in \mathbb{F}^{n}$ of $\mathbf{h}$ under the affine map $\mathcal{S}$.

2. Lift $\mathbf{x}$ to the extension field $\mathbb{E}$ (using the isomorphism $\Phi$ ). Denote the result by $X$.

Choose random values for the vinegar variables $v_{1}, \ldots, v_{v} \in \mathbb{F}$ and compute $\mathcal{F}_{V}=\mathcal{F}\left(v_{1}, \ldots, v_{v}\right)$.

Solve the univariate polynomial equation $\mathcal{F}_{V}(Y)=X$ by Berlekamp's algorithm and compute $\mathbf{y}^{\prime}=\Phi^{-1}(Y) \in \mathbb{F}^{n}$.

Set $\mathbf{y}=\left(\mathbf{y}^{\prime}|| v_{1}\|\ldots\| v_{v}\right)$.

3. Compute the signature $\mathbf{z} \in \mathbb{F}^{n+v}$ by $\mathbf{z}=\mathcal{T}^{-1}(\mathbf{y})$.

Signature verification: To check the authenticity of a signature $\mathbf{z} \in \mathbb{F}^{n+v}$, one simply computes $\mathbf{h}^{\prime}=\mathcal{P}(\mathbf{z}) \in \mathbb{F}^{n-a}$. If $\mathbf{h}^{\prime}=\mathbf{h}$ holds, the signature is accepted, otherwise rejected. 


\subsection{QUARTZ}

In 2001, Patarin and Courtois proposed the multivariate signature scheme QUARTZ [21], which is based on the concept of HFEv-. Indeed, the public and private maps of QUARTZ are HFEv- maps with the parameters

$$
(\mathbb{F}, n, D, a, v)=(\mathrm{GF}(2), 103,129,3,4) .
$$

Due to this choice, the public key $\mathcal{P}$ of QUARTZ is a quadratic map from $\mathbb{F}^{107}$ to $\mathbb{F}^{100}$. The public key size of QUARTZ is $71 \mathrm{kB}$, the private key size $3 \mathrm{kB}$.

The input length of QUARTZ is only $n-a=100$ bit. Therefore, it is possible for an attacker to use a birthday attack to find two different messages $m_{1}$ and $m_{2}$ which map to the same input value $\mathbf{h} \in \mathbb{F}^{100}$ and therefore to the same signature.

To prevent this kind of attack, Patarin and Courtois developed a special procedure for the signature generation process of QUARTZ. Roughly spoken, one computes four HFEv- signatures (for the messages $\mathbf{h}, \mathcal{H}(\mathbf{h} \| 0 x 00), \mathcal{H}(\mathbf{h} \| 0 x 01$ ) and $\mathcal{H}(\mathbf{h}|| 0 x 02))$ and combines them to a single 128 bit signature of the message $\mathbf{h}$. Analogously, during the signature verification process, one has to use the public key $\mathcal{P}$ four times.

\subsection{Performance}

The most costly step during the signature generation process of HFEv- based signature schemes such as QUARTZ is the inversion of the univariate polynomial equation $\mathcal{F}_{V}$ over the extension field $\mathbb{E}$. This step is usually performed by Berlekamp's algorithm, whose complexity can be estimated by [24]

$$
\mathcal{O}\left(D^{3}+n \cdot D^{2}\right) .
$$

As can be seen from equation (6), the complexity of inverting $\mathcal{F}_{V}$ and therefore of the signature generation process of HFEv- based schemes is mainly determined by the degree $D$ of the HFE polynomial. Due to the high degree of the HFE polynomial used in QUARTZ, the inversion of $\mathcal{F}_{V}$ is very costly. Furthermore, we have to perform this step four times during the signature generation of QUARTZ. Additionally, the design of QUARTZ requires the central equation $\mathcal{F}_{V}(Y)=X$ to have a unique root. Since, after choosing random values for Minus equations and Vinegar variables, $\mathcal{F}_{V}$ can be seen as a random function, this happens with probability about $\frac{1}{e}$. Altogether, we therefore have to run Berlekamp's algorithm about $4 \cdot e$ times during the signature generation process of QUARTZ. Thus, the

QUARTZ signature scheme is rather slow and it takes about 11 seconds to generate a signature [5].

\subsection{Security of HFEv- based schemes}

The most important attacks against signature schemes of the HFEv- type are

- the MinRank attack and

- direct algebraic attacks. 
The MinRank attack on HFE In this paragraph we describe the attack of Kipnis and Shamir [18] against the HFE cryptosystem. For the simplicity of our description we restrict ourselves to homogeneous maps $\mathcal{F}$ and $\mathcal{P}$.

The key idea of the attack is to lift the maps $\mathcal{S}, \mathcal{T}$ and $\mathcal{P}$ to functions $\mathcal{S}^{\star}, \mathcal{T}^{\star}$ and $\mathcal{P}^{\star}$ over the extension field $\mathbb{E}$. Since $\mathcal{S}$ and $\mathcal{T}$ are linear maps, $\mathcal{S}^{\star}$ and $\mathcal{T}^{\star}$ have the form

$$
\mathcal{S}^{\star}(X)=\sum_{i=1}^{n-1} s_{i} \cdot X^{q^{i}} \text { and } \mathcal{T}^{\star}(X)=\sum_{i=1}^{n-1} t_{i} \cdot X^{q^{i}},
$$

with coefficients $s_{i}$ and $t_{i} \in \mathbb{E}$. The function $\mathcal{P}^{\star}$ can be expressed as

$$
\mathcal{P}^{\star}(X)=\sum_{i=0}^{n-1} \sum_{j=0}^{n-1} p_{i j}^{\star} X^{q^{i}+q^{j}}=\underline{X} \cdot P^{\star} \cdot \underline{X}^{T},
$$

where $P^{\star}=\left[p_{i j}^{\star}\right]$ and $\underline{X}=\left(X^{q^{0}}, X^{q^{1}}, \ldots, X^{q^{n-1}}\right)$. Due to the relation $\mathcal{P}^{\star}(X)=$ $\mathcal{S}^{\star} \circ \mathcal{F} \circ \mathcal{T}^{\star}(X)$ we get $\mathcal{S}^{\star-1} \circ \mathcal{P}^{\star}(X)=\mathcal{F} \circ \mathcal{T}^{\star}(X)$ and

$$
\tilde{P}=\sum_{k=0}^{n-1} s_{k} \cdot G^{\star k}=W \cdot F \cdot W^{T}
$$

with $g_{i j}^{\star}{ }^{k}=\left(p^{\star}{ }_{i-k} \bmod n, j-k \bmod n\right)^{q^{k}}, w_{i j}=s_{j-i \bmod n}^{q^{i}}$ and $F$ being the $n \times n$ matrix representing the central map $\mathcal{F}$. Note that, due to the special structure of $\mathcal{F}$, the only non zero entries in the matrix $F$ are located in the upper left $r \times r$ submatrix $\left(r=\left\lfloor\log _{q} D-1\right\rfloor+1\right)$.

Therefore, the rank of the matrix $W \cdot F \cdot W^{T}$ is less or equal to $r$, which means that we can determine the coefficients $s_{k}$ of equation (9) by solving an instance of the MinRank problem.

In the setting of HFEv-, the rank of this matrix can, for odd characteristic, be bounded from above by [11]

$$
\operatorname{Rank}(\widetilde{P}) \leq r+a+v .
$$

Under the assumption that the vinegar maps $\beta_{i}$ look like random functions, we find that this bound is tight.

For fields of even characteristic we eventually have to decrease this rank by 1 , since over those fields, the matrix $\widetilde{P}$ is always of even rank. The complexity of the MinRank attack against HFEv- based schemes is therefore given roughly by

$$
\text { Complexity }_{\text {MinRank }}=\mathcal{O}\left(q^{n \cdot(r+v+a-1)} \cdot(n-a)^{3}\right) .
$$

Direct attacks For the HFE family of schemes, the direct attack, namely the attack by directly solving the public equation $\mathcal{P}(\mathbf{x})=\mathbf{h}$ by an algorithm like XL or a Gröbner basis method such as $F_{4}[12]$ is a major concern due to which happened to HFE challenge 1. At the time of the design of QUARTZ, very little was 
known theoretically about the complexity of algebraic attacks against the HFE family of systems, in particular, the HFEv- schemes. The authors of QUARTZ did not actually give an explanation for their selection of the parameters and therefore the parameter selection of their scheme was not supported by theoretical results. We need to point out that, as has been shown by experiments [19], the public systems of HFEv- based schemes can be solved easier than random systems.

Recently, there has been a fundamental breakthrough in terms of understanding how algebraic attacks on the HFE family of systems work $[8,9,11]$. In particular, we now have a solid insight what happens in the case of HFEv-. An upper bound for the degeneration degree of a Gröbner Basis attack against HFEv- systems is given by [11]

$$
\mathrm{d}_{\mathrm{reg}} \leq \begin{cases}\frac{(q-1) \cdot(r-1+a+v)}{2}+2 & q \text { even and } r+a \text { odd } \\ \frac{(q-1) \cdot(r+a+v)}{2}+2 & \text { otherwise }\end{cases}
$$

where $r$ is given by $r=\left\lfloor\log _{q}(D-1)\right\rfloor+1$.

Note: In [6] Courtois et al. estimated the complexity of a direct attack on QUARTZ by $2^{74}$ operations. However, they underestimated the degree of regularity of solving an HFEv- system drastically.

\section{Design principles for HFEv- based signature schemes}

The theoretical breakthrough mentioned in the previous subsection indicates that it might be possible to substantially improve the original design of QUARTZ without reducing the security of the scheme, if we adapt the number of Minus equations and Vinegar variables in an appropriate way. By reducing the degree of the central HFEv- polynomial we can speed up the operations of Berlekamp's algorithm and therefore the signature generation process of the HFEv- scheme. In this section, we analyze by experiments the behavior of direct attacks against HFEv- schemes and the tightness of the upper bound given by equation (12). From our results we derive design principles for the construction of our new HFEv- based signature scheme Gui presented in the next section.

In particular, we answer in this section the following questions.

1. Equation (12) shows a tradeoff between the degree $D$ of the HFE polynomial and the sum $a+v$ of minus equations and vinegar variables. This would enable us to use low degree HFE polynomials in the construction of HFEv- based signature schemes and therefore to improve their performance drastically. Can we verify this by experiments?

2. Is the ratio between $a$ and $v$ important for the security of the scheme?

3 . Is the upper bound on the degree of regularity given by equation (12) reasonably tight?

To answer these questions, we performed a large number of experiments with the $F_{4}$ algorithm integrated in MAGMA. As we found, adding the field equations 
$x_{i}^{2}-x_{i}$ to the system makes a huge difference regarding the degree of regularity and the running time of the system.

\subsection{Can we use HFE polynomials of low degree D?}

To improve the efficiency of the signature generation process we are interested in decreasing the degree of the HFE polynomial in use as far as possible without weakening the security of the scheme. Doing so will reduce the complexity of Berlekamp's algorithm (see equation (6)) and therefore improve the performance of our scheme significantly. So, the first question we have to answer in this context is the following.

Which value $D$ should we choose to obtain secure and efficient HFEvbased schemes?

- $D=2,3$ : Such small values of $D$ would lead to matrices $F$ of rank 2 . We therefore do not think that these schemes can be secure.

$-D=5$ : Although the plain HFE scheme with an HFE polynomial of degree $5(r=3)$ is highly insecure, we believe that the modificated HFEv- scheme provides adequate security.

- $D=9,17$ : Other promising values for the degree of the HFE polynomial in use are $D=9$ and $D=17$, which lead to values of $r$ of 4 and 5 respectively.

In the first row of experiments we analyzed the behavior of direct attacks against HFEv- systems over GF(2) with different values of $D$. For this, we fixed the number $n$ of equations in the system. For different values of $D, a$ and $v$ we created HFEv- systems and fixed $a+v$ variables randomly to get determined systems. After adding the field equations $x_{i}^{2}-x_{i}$ we solved the systems using MAGMA's implementation of the $F_{4}$ algorithm. Table 1 shows the results of our experiments with determined HFEv- systems of 20 and 25 equations respectively. The degree of regularity of a random system of this size is 5 and 6 respectively. The table shows, for different values of $D$, the minimal values of $a$ and $v$ needed to reach this degree. Although, because of memory restrictions, we could not perform our experiments for larger values of $n$, we expect that similar results hold for arbitrary numbers of equations.

From the above experiments we obtain the following important observation

Let $d_{r e g}$ be the degree of regularity of a direct attack against an HFEv- system with parameters $D_{1}, n, a_{1}, v_{1}$ and let $D_{2}<D_{1}$.

By choosing large enough values for $a_{2}$ and $v_{2}$, we can obtain an HFEvscheme with parameters $D_{2}, n, a_{2}, v_{2}$, such that the degree of regularity of a direct attack against this system is $d_{r e g}$, too. 


\begin{tabular}{|c|c|c|c|c|c||c|c|c|c|}
\hline & & \multicolumn{4}{|c|}{20 equations } & \multicolumn{4}{c|}{25 equations } \\
\hline $\mathrm{D}$ & $\mathrm{r}$ & minimal a,v & $d_{\text {reg }}$ & time $(\mathrm{s})$ & memory $(\mathrm{MB})$ & minimal a,v & $d_{\text {reg }}$ & time $(\mathrm{s})$ & memory $(\mathrm{MB})$ \\
\hline 129 & 8 & $a=v=0$ & 5 & 2.74 & 109.7 & $a=v=1$ & 6 & 286.2 & 8,021 \\
\hline 65 & 7 & $a=0, v=1$ & 5 & 2.69 & 110.7 & $a=v=2$ & 6 & 276.0 & 7,781 \\
\hline 33 & 6 & $a=v=1$ & 5 & 2.75 & 109.7 & $a=2, v=3$ & 6 & 260.4 & 7,762 \\
\hline 17 & 5 & $a=1, v=2$ & 5 & 2.72 & 109.7 & $a=v=3$ & 6 & 258.7 & 7,751 \\
\hline 9 & 4 & $a=v=2$ & 5 & 2.73 & 110.7 & $a=3, v=4$ & 6 & 266.4 & 7,693 \\
\hline 5 & 3 & $a=2, v=3$ & 5 & 2.73 & 109.6 & $a=v=4$ & 6 & 262.8 & 7,580 \\
\hline \hline
\end{tabular}

Table 1. Experiments with $F_{4}$ on determined HFEv- systems with 20 and 25 equations

From this observation we derive our first design principle for the construction of HFEv- based signature schemes.

Design Principle 1: For the construction of HFEv- based signature schemes we use for efficiency reasons HFE polynomials of small degree $D$, namely $D \in\{5,9,17\}$. We then increase the numbers of Minus equations $a$ and Vinegar variables $v$ to obtain a secure scheme.

\subsection{Is the concrete choice of $a$ and $v$ important for the security of the scheme?}

To answer this question, we performed experiments of the following type. For a fixed degree $D$ of the HFE polynomial, a fixed number of equations and a fixed value $s$ we created HFEv- systems with $a \in\{0, \ldots, s\}$ and $v=s-a$. After fixing $n-a$ variables to get a determined system and adding the field equations $x_{i}^{2}-x_{i}$ we solved the systems by the $F_{4}$ algorithm integrated in MAGMA. The results of these experiments are shown in Table 2 and 3.

\begin{tabular}{|c|c|c|c|c|}
\hline$a$ & $v$ & $d_{\text {reg }}$ & time $(\mathrm{s})$ & memory (MB) \\
\hline 0 & 4 & 5 & 2.77 & 109.7 \\
\hline 1 & 3 & 5 & 2.78 & 110.8 \\
\hline 2 & 2 & 5 & 2.73 & 110.7 \\
\hline 3 & 1 & 5 & 2.73 & 110.8 \\
\hline 4 & 0 & 5 & 2.79 & 108.7 \\
\hline
\end{tabular}

Table 2. Experiments with $F_{4}$ on determined HFEv- systems with $D=9,20$ equations and $a+v=4$

As Tables 2 and 3 show, the concrete choice of $a$ and $v$ only plays a minor role for the complexity of direct attacks against HFEv- schemes, as long as their sum stays fixed. However, as the last row of Table 3 shows, it might not be a 


\begin{tabular}{|c|c|c|c|c|}
\hline$a$ & $v$ & $d_{\text {reg }}$ & time (s) & memory (MB) \\
\hline 0 & 7 & 6 & 248.9 & 7,582 \\
\hline 1 & 6 & 6 & 247.4 & 7,582 \\
\hline 2 & 5 & 6 & 258.0 & 7,580 \\
\hline 3 & 4 & 6 & 266.4 & 7,693 \\
\hline 4 & 3 & 6 & 258.3 & 7,578 \\
\hline 5 & 2 & 6 & 248.5 & 7,579 \\
\hline 6 & 1 & 6 & 253.3 & 7,581 \\
\hline 7 & 0 & $\mathbf{5}$ & 99.5 & 1,380 \\
\hline
\end{tabular}

Table 3. Experiments with $F_{4}$ on determined HFEv- systems with $D=9,25$ equations and $a+v=7$

good choice to choose $v$ to be zero. From this observation we derive our second design principle for HFEv- based signature schemes.

Design Principle 2: In the design of HFEv- based signature schemes we choose the number of minus equations $a$ and the number of vinegar variables $v$ to be as equal as possible, i.e. $v-a \leq 1$.

\subsection{Is the upper bound on $\boldsymbol{d}_{r e g}$ given by equation (12) reasonably tight?}

In this section we check by experiments if the upper bound on the degree of regularity given by equation (12) is tight. Due to memory restrictions, we can show the tightness of equation (12) only for some small values of $D, a$ and $v$. However, for all values of $D$ used in our scheme Gui $(D \in\{5,9,17\}$ we could find parameter sets for which the bound (12) is tight (see Table 4).

\begin{tabular}{|c|c|c|c|cc|}
\hline $\mathrm{D}$ & $\mathrm{a}$ & $\mathrm{v}$ & upper bound for $d_{\text {reg }}(12)$ & $d_{\text {reg }}$ (experimental) \\
\hline \multirow{2}{*}{5} & 0 & 0 & 3 & 3 & for $n \geq 10$ \\
\cline { 2 - 5 } & 1 & 1 & 4 & 4 & for $n \geq 23$ \\
\hline \multirow{2}{*}{9} & 0 & 1 & 4 & 4 & for $n \geq 23$ \\
\cline { 2 - 5 } & 1 & 1 & 4 & 4 & for $n \geq 21$ \\
\hline \multirow{2}{*}{17} & 0 & 0 & 4 & 4 & for $n \geq 15$ \\
\cline { 2 - 6 } & 0 & 1 & 4 & 4 & for $n \geq 12$ \\
\hline
\end{tabular}

Table 4. Parameter sets, for which the upper bound (12) is tight

For most of the other parameter sets, we missed the upper bound on the degree of regularity given by equation (12) by at most 1 . We believe that, by increasing the number of equations in the systems, it would be possible to reach the upper bound for arbitrary values of $(D, a, v)$. However, due to memory restrictions, 
we could not perform experiments with more than 38 equations. The degree of regularity of a system with the $n$ random quadratic equations in $n$ binary variables is also bounded by

$$
\mathrm{d}_{\mathrm{reg}} \leq \min \left\{D: \text { the coefficient of } t^{D} \text { in }\left[(1+t)^{n}\left(1+t^{2}\right)^{-n}\right] \text { is nonpositive }\right\} \text {, }
$$

by standard references (e.g. [1]). The degree of regularity of 40 random binary quadratic equations in 40 variables is only 7 . Therefore the upper bound of (12), if more than 7, is not testable at $n=38$. We do show (Table 5) that for all of our proposed values of $D$ a degree of regularity of at least 7 is reached. These results are the basis of our parameter choice for Gui.

Figures 2 to 4 show the degree of regularity of the $F_{4}$ algorithm on determined

\begin{tabular}{|c|c|c|cr|c|}
\hline $\mathrm{D}$ & $\mathrm{a}$ & $\mathrm{v}$ & $d_{\text {reg }}$ (experimental) & upper bound for $d_{\text {reg }}(12)$ \\
\hline 5 & 6 & 6 & 7 & for $n \geq 38$ & 9 \\
\hline 9 & 5 & 5 & 7 & for $n \geq 37$ & 8 \\
\hline 17 & 4 & 4 & 7 & for $n \geq 37$ & 8 \\
\hline
\end{tabular}

Table 5. Parameter sets which lead to $d_{r e g} \geq 7$

HFEv- systems over $\operatorname{GF}(2)$ with $D \in\{5,9,17\}$ and $a=v \in\{0, \ldots, 5\}$.

\section{The New Multivariate Signature Scheme Gui}

Based on our experiments presented in the previous section we propose three different versions of our HFEv- based signature scheme Gui over the field GF(2):

- Gui-96 with $(n, D, a, v)=(96,5,6,6)$ with 90 equations in 102 variables,

- Gui-95 with $(n, D, a, v)=(95,9,5,5)$ with 90 equations in 100 variables and

- Gui-94 with $(n, D, a, v)=(94,17,4,4)$ with 90 equations in 98 variables.

The complexity of direct attacks against these schemes can be estimated as follows.

According to our experiments (see Table 5), the degree of regularity of the $F_{4}$ algorithm against these schemes will be at least 7 .

The number $T$ of top-level monomials in the solving step of the $F_{4}$ algorithm is therefore given by

$$
T=\left(\begin{array}{c}
n-a \\
d_{\text {reg }}
\end{array}\right) \geq\left(\begin{array}{c}
90 \\
7
\end{array}\right) \geq 2^{33.6}
$$

The number of non zero elements in each row can be estimated by $\tau=\left(\begin{array}{c}n-a \\ 2\end{array}\right)>$ $2^{12}$. Therefore we get for the complexity of a direct attack against one of our schemes

$$
\text { Complexity }_{F_{4} / F_{5}} \geq 3 \cdot \tau \cdot T^{2}>2^{80.7} .
$$

Note that this number is very optimistic since we assume that the degree of regularity will not rise above 7 . 


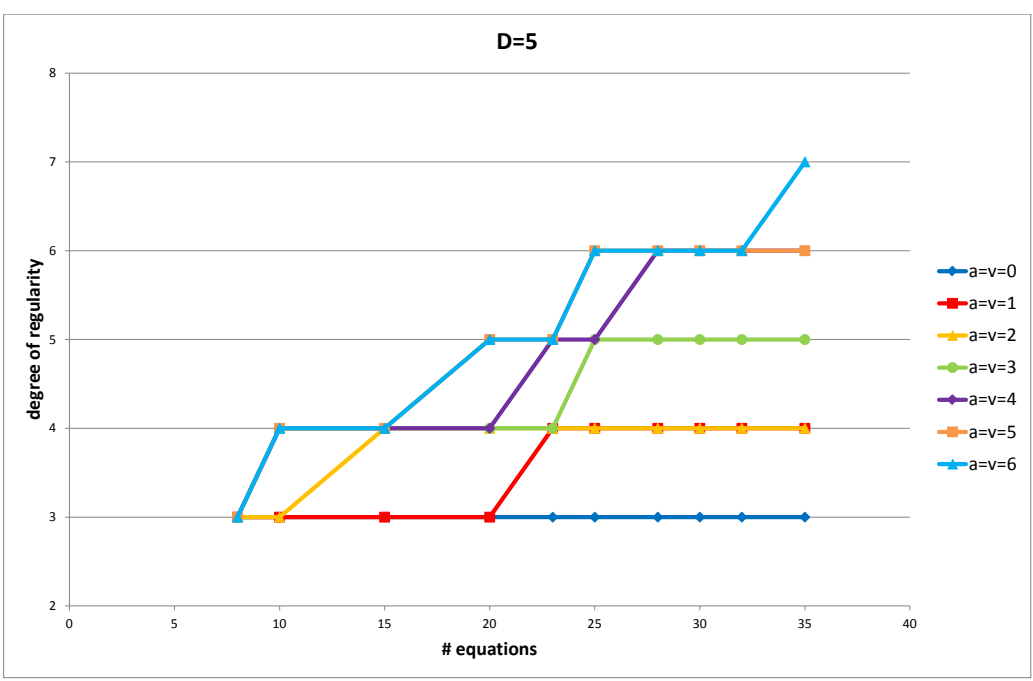

Fig. 2. Degree of regularity of determined HFEv- systems $(D=5)$

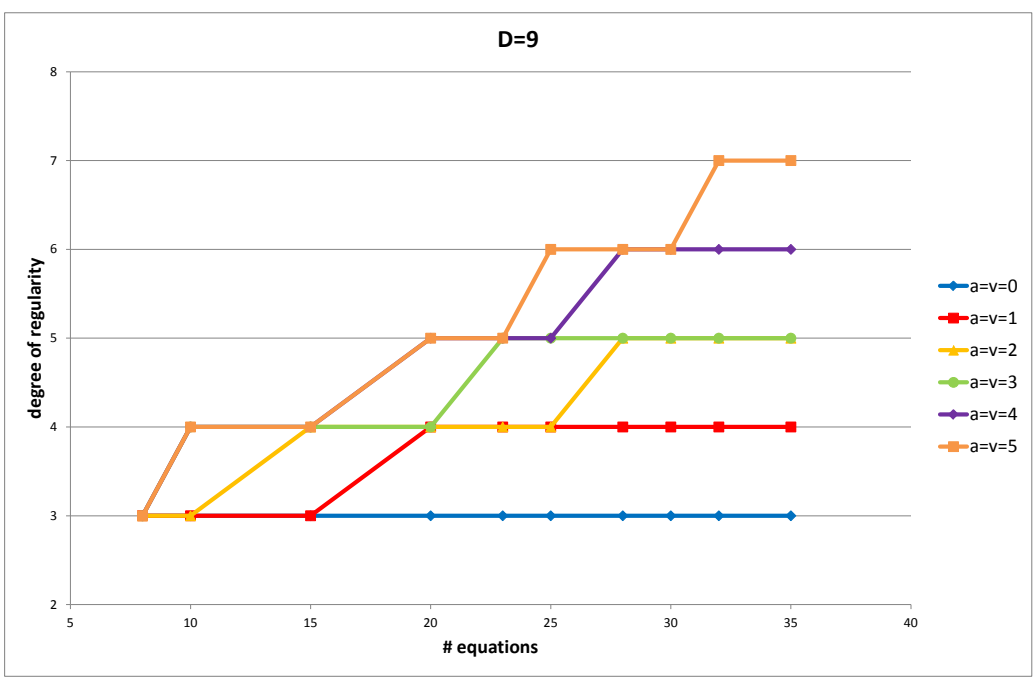

Fig. 3. Degree of regularity of determined HFEv- systems $(D=9)$ 


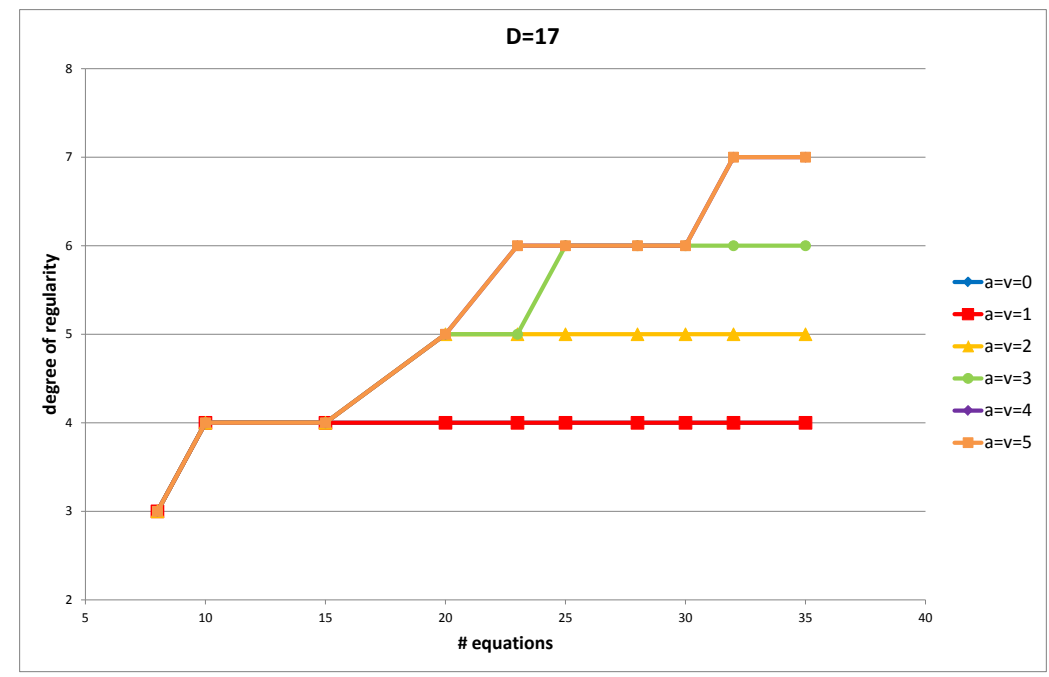

Fig. 4. Degree of regularity of determined HFEv- systems $(D=17)$

Additionally, for better comparison to standard signature schemes, we propose a fourth version of Gui, Gui-127, with the parameters $(n, D, a, v)=(127,9,4,6)$, providing a security level of 120 bits.

\subsection{Signature Generation}

The central component of the signature generation process of Gui is inverting the HFEv- core map.

To compute a pre-image of a $(n-a)$ bit digest $\mathbf{h}$, one first has to choose random values for the Minus equations and the Vinegar variables. In our concrete implementation, these values are the last $a+v$ bits of SHA-256(h). After that, one computes recursively $\mathbf{x}=\mathcal{S}^{-1}(\mathbf{h}), \mathbf{y}=\mathcal{F}_{V}^{-1}(\mathbf{x})$ and $\mathbf{z}=\mathcal{T}^{-1}(\mathbf{y})$ (see Figure $5)$.

For the parameters of Gui, the length of the digest $\mathbf{h}$ is only $n-a=90$ bits. To prevent birthday attacks, we therefore have to perform the above process several times (for different values of $\mathbf{h}$ ). We denote this repetition factor by $k$ and set $k=3$ for Gui-96 and Gui-95. For Gui-94 and Gui-127 the value $k$ is chosen to be 4 . 
The signature generation process of Gui works as shown in Algorithm 1 and Figure 6.

We initialize the $n-a$ vector $S_{0}$ to be $\mathbf{0}$ and compute the SHA-256 hash value $\mathbf{h}$ of the message. Let $D_{1}$ be the bitstring consisting of the first $(n-a)$ bits of h. We compute the pre-image of $D_{1}$ under the HFEv- core (see above) and split the result into an $(n-a)$ bit string $S_{1}$ and an $a+v$ bit string $X_{1}$.

We set $D_{2}$ to be the string consisting of the first $(n-a)$ bits of SHA-256(h) and compute the HFEv- pre-image of $D_{2} \oplus S_{1}$. Again, the result is split into the two parts $S_{2}(n-a$ bits $)$ and $X_{2}(a+v$ bits $)$. This process is repeated, until we have values $S_{i}, X_{i}$ for $i=1, \ldots, k$.

The final signature of the message is given by $\sigma=\left(S_{k}\left\|X_{k}\right\| \ldots \| X_{1}\right)$. The resulting signature sizes for our schemes can be found in Table 6 .

A detailed description, how the inversion of the central HFEv- map is performed in our implementation, can be found in Section 6.2. Due to some flaws in the SHA-1 algorithm, we replace the SHA-1 hash function used in the original QUARTZ design by SHA-256.

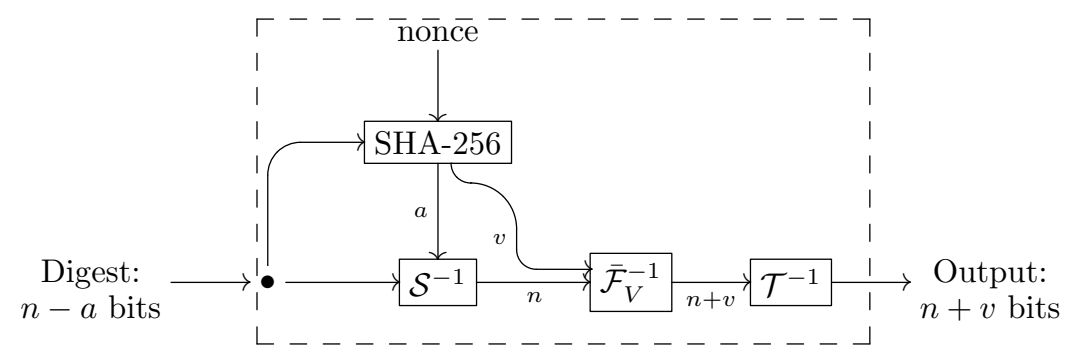

Fig. 5. Core operations of HFEv-

\subsection{Signature Verification}

To check the authenticity of a signature $\sigma \in \mathrm{GF}(2)^{(n-a)+k(a+v)}$ we parse $\sigma$ into $S_{k}, X_{k}, \ldots, X_{1}$ and compute $D_{1}, \ldots, D_{k}$ as shown in Section 5.1. For $i=k-1$ to 0 we compute recursively $S_{i}=\mathcal{P}\left(S_{i+1} \| X_{i+1}\right) \oplus D_{i+1}$. The signature is accepted, if and only if $S_{0}=\mathbf{0}$ holds.

By the above construction of the signature generation and verification process we prevent birthday attacks as follows. We consider an adversary $A$ who wants to find two messages $m_{1}$ and $m_{2}$ which lead to the same signature $\sigma$. 

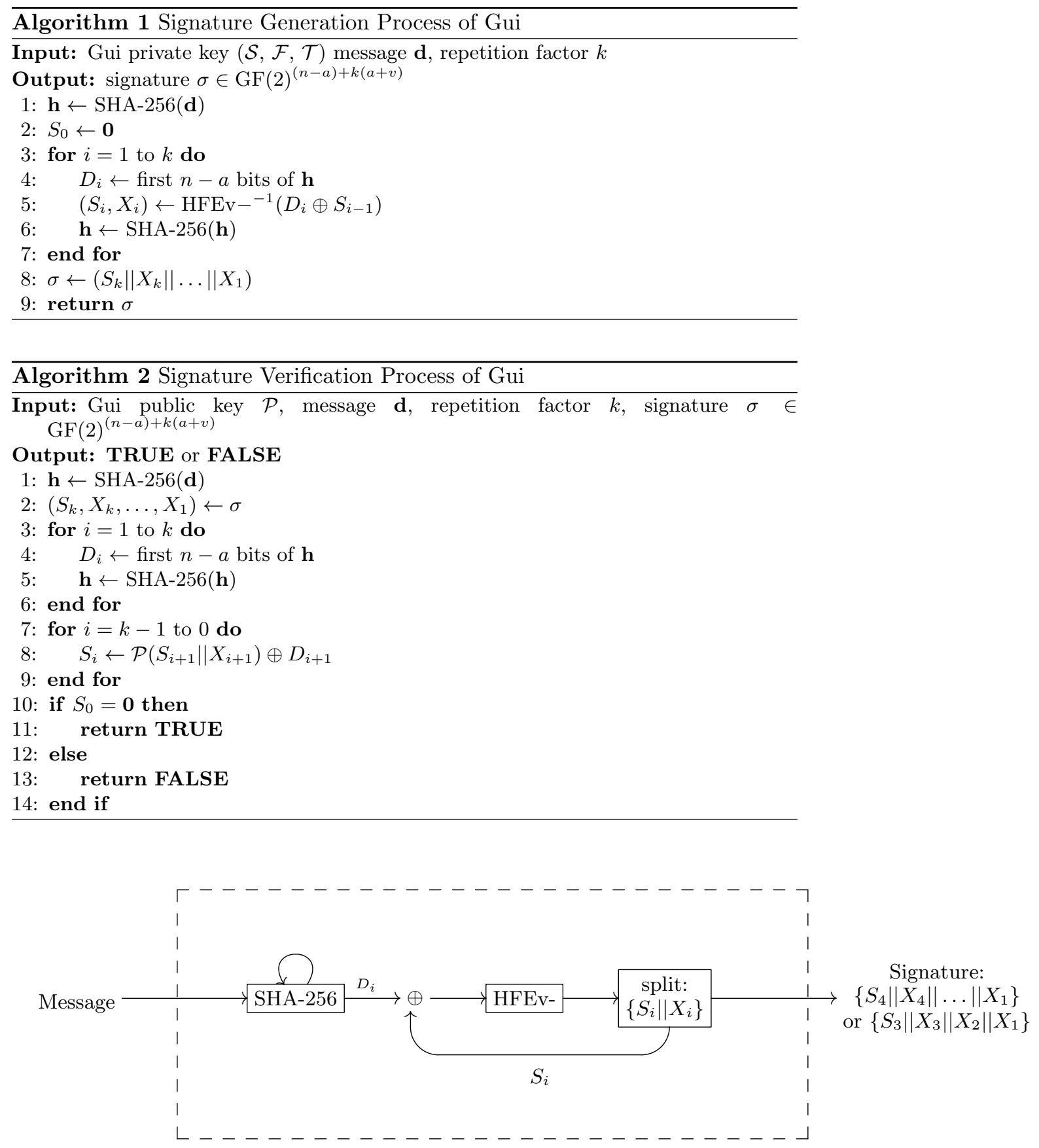

Fig. 6. Signature Generation Process of Gui 
In the context of the plain HFEv- signature scheme it would be enough to find two messages $m_{1}$ and $m_{2}$ such that SHA-256 $\left(m_{1}\right)_{i}=$ SHA-256 $\left(m_{2}\right)_{i}$ for the first $n-a$ bits. If $(n-a) \leq 160$, the adversary can find $m_{1}$ and $m_{2}$ by a birthday attack.

In the context of our scheme Gui, the adversary now has to find messages $m_{1}$ and $m_{2}$ which lead to the same values of $D_{1}, \ldots, D_{k}$. For our values of the repetition factor $k$, this corresponds to finding a collision for a hash function of length 270, 360 and 492 bit (Gui-95, Gui-94 and Gui-127 respectively). This is, in general, assumed to be infeasible.

\begin{tabular}{|c|c|r|r||r|c|}
\hline scheme & $\begin{array}{c}\text { core map } \\
\text { HFEv-(n,D,a,v) }\end{array}$ & $\begin{array}{c}\text { public key } \\
\text { size (byte) }\end{array}$ & $\begin{array}{c}\text { private key } \\
\text { size (byte) }\end{array}$ & $\begin{array}{c}\text { repetition factor } \\
k\end{array}$ & $\begin{array}{c}\text { signature } \\
\text { size (bit) }\end{array}$ \\
\hline Gui-96 & $(96,5,6,6)$ & 63036 & 3175 & 3 & 126 \\
\hline Gui-95 & $(95,9,5,5)$ & 60600 & 3053 & 3 & 120 \\
\hline Gui-94 & $(94,17,4,4)$ & 58212 & 2943 & 4 & 122 \\
\hline \hline Gui-127 & $(127,9,4,6)$ & 142576 & 5350 & 4 & 163 \\
\hline \hline Quartz & $(103,129,3,4)$ & 75515 & 3774 & 4 & 128 \\
\hline
\end{tabular}

Table 6. Key and signature sizes of Gui-94,Gui-95,Gui-96, and Gui-127

\section{Implementation and Comparison}

In this section we present the details of our implementation of the Gui signature scheme and compare the performance of our scheme with that of the original QUARTZ and other standard signature schemes.

\subsection{Arithmetics over Finite Fields}

The first step in our implementation of the Gui signature scheme is to provide efficient arithmetics over the large binary fields in use. To speed up these computations, we use a set of new processor instructions for carry-less multiplication: PCLMULQDQ [27].

The instruction set PCLMULQDQ allows the efficient multiplication of two 64bit polynomials over GF(2) resulting in an 128-bit polynomial. The PCLMULQDQ instructions are available on some new processors of Intel and AMD. Performance data of PCLMULQDQ can be found in Table 7.

In the case of Gui, the extension field $\mathbb{E}$ has less than $2^{128}$ elements. We represent an element of the field $\mathbb{E}$ as a polynomial over $\mathrm{GF}(2)$ which can be divided into two 64-bits polynomials. 


\begin{tabular}{|c|c|c|c|}
\hline \multicolumn{2}{|c|}{$\begin{array}{c}\text { processor } \\
\text { type }\end{array}$} & $\begin{array}{c}\text { latency } \\
\text { cycles }\end{array}$ & $\begin{array}{c}\text { throughput } \\
\text { cycles/multiplication }\end{array}$ \\
\hline \multirow{3}{*}{ Intel } & Sandy Bridge & 14 & 8 \\
\cline { 2 - 4 } & Ivy Bridge & 14 & 8 \\
\cline { 2 - 4 } & Hashwell & 7 & 2 \\
\hline \hline \multirow{3}{*}{ AMD } & Bulldozer & 12 & 7 \\
\cline { 2 - 4 } & Piledriver & 12 & 7 \\
\cline { 2 - 4 } & Steamroller & 11 & 7 \\
\hline
\end{tabular}

Table 7. Performance of PCLMULQDQ on different platforms (source: [15], [13])

A multiplication over the large field $\mathbb{E}$ is divided into two phases, namely a multiplication and a reduction phase.

In the multiplication phase, the multiplication of two 128-bit polynomials can be performed by 4 calls of PCLMULQDQ. With the help of the Karatsuba algorithm, we can avoid one call of PCLMULQDQ and therefore its long latency (see Table 7). To compute the square of one element of $\mathbb{E}$, we need only two calls of PCLMULQDQ since we are operating in a field of characteristic 2 .

The reduction phase of the field multiplication heavily depends on the field representation. For the original QUARTZ $\left(\mathrm{GF}\left(2^{103}\right)\right)$ the authors used $\operatorname{GF}\left(2^{103}\right):=$ $\mathrm{GF}(2)[x] /\left(x^{103}+x^{9}+1\right)[21]$. For Gui, we choose the field representations

$-\mathrm{GF}\left(2^{94}\right):=\mathrm{GF}(2)[x] /\left(x^{94}+x^{21}+1\right)$,

$-\mathrm{GF}\left(2^{95}\right):=\mathrm{GF}(2)[x] /\left(x^{95}+x^{11}+1\right)$,

$-\mathrm{GF}\left(2^{96}\right):=\mathrm{GF}(2)[x] /\left(x^{96}+x^{10}+x^{9}+x^{6}+1\right)$ and

$-\mathrm{GF}\left(2^{127}\right):=\mathrm{GF}(2)[x] /\left(x^{127}+x+1\right)$ respectively.

The baseline for the reduction phase is two calls of PCLMULQDQ since, after the multiplication phase, the degree of the polynomial will be greater than $2 \times 64$. The irreducible polynomials above are chosen to contain only few terms of low degree. With few terms in the irreducible polynomials, we may replace the use of PCLMULQDQ by a few logic shifts and XOR instructions.

In the $\operatorname{GF}\left(2^{127}\right)$ case, for example, the reduction can be performed by only two 128-bit shifts for the $x^{128}$ part and one conditional XOR for the $x^{127}$ term, avoiding at least two calls of PCLMULQDQ while reducing the high 128 bit register.

Another technique is to represent elements as 128-bit polynomials while avoiding full reduction. This allows us to perform the reduction of degree 128-191 and 192-255 terms using only two calls of PCLMULQDQ without data dependency. In the $\mathrm{GF}\left(2^{96}\right)$ case, for example, we can perform the reduction phase by multiplying the degree 128-191 terms by $x^{128}=x^{42}+x^{41}+x^{38}+x^{32}$ and the degree 192-255 terms with $x^{192}=x^{20}+x^{18}+x^{12}+1$. All the polynomials in use have degree $\leq 64$, and we can perform the reduction by two calls of PCLMULQDQ. 


\subsection{Inverting the HFEv- core}

In this section we describe how we can perform the inversion of the central HFEv- equation $\mathcal{F}_{V}(Y)=X$ efficiently. During the signature generation process of Gui we have to perform this step several times to avoid birthday attacks (see Section 5.1). Therefore it is extremely important to perform this step efficiently.

To invert the central HFEv- equation, we have to perform Berlekamp's algorithm to find the roots of the polynomial $\mathcal{F}_{V}(Y)-X$. Since the design of QUARTZ and Gui requires $\mathcal{F}_{V}(Y)-X$ to have a unique solution, we only have to perform the first step of Berlekamp's algorithm, i.e. the computation of

$$
\operatorname{gcd}\left(\mathcal{F}_{V}(Y)-X, Y^{2^{n}}-Y\right)
$$

We have

$$
\begin{aligned}
& \operatorname{gcd}\left(\mathcal{F}_{V}(Y)-X, Y^{2^{n}}-Y\right) \\
= & \operatorname{gcd}\left(\mathcal{F}_{V}(Y)-X, \prod_{i \in \mathbb{F}_{2^{n}}, i \neq 0}(Y-i)\right) \\
= & \prod_{i: \mathcal{F}_{V}(i)=X}(Y-i) .
\end{aligned}
$$

Therefore the main process in creating a signature consists in computing $\operatorname{gcd}\left(\mathcal{F}_{V}(Y)-X, Y^{2^{n}}-Y\right.$ ). The number of roots of $\mathcal{F}_{V}(Y)-X$ (as well as the only solution when that happens) can obviously be read off from the result.

Probability of a unique root Every time we choose the values of Minus equations and Vinegar variables, we basically pick a random central equation $\mathcal{F}(Y)-X=0$. The probability of this equation having a unique solution is about $1 / e$. Therefore, in order to invert the HFEv- central equation, we have to perform the gcd computation about $e$ times.

How do we optimize the computation of the gcd? The main computation consumption in this step comes from the division of the extreme high power polynomial $Y^{2^{n}}-Y \bmod \mathcal{F}_{V}(Y)$. A naive long division is unacceptable for this purpose due to its slow reduction pace. Instead of this, we choose to recursively raise the lower degree polynomial $Y^{2^{m}}$ to the power of 2 .

$$
\begin{aligned}
& \left(Y^{2^{m}} \bmod \mathcal{F}_{V}(Y)\right)^{2} \bmod \mathcal{F}_{V}(Y) \\
= & \left(\sum_{i<2^{m}} b_{i} Y^{i}\right)^{2} \bmod \mathcal{F}_{V}(Y) \\
= & \left(\sum_{i<2^{m}} b_{i}^{2} Y^{2 i}\right) \bmod \mathcal{F}_{V}(Y)
\end{aligned}
$$

By multiplying $Y$ to the naive relation $Y^{D}=\sum_{0 \leq i \leq j, 2^{i}+2^{j}<D} a_{i j} Y^{2^{i}+2^{j}}$, we can prepare a table for $Y^{2 i} \bmod \mathcal{F}_{V}(Y)$ first. The rest of computation of the raising 
process is to square all the coefficients $b_{i}$ in $Y^{2^{m}} \bmod \mathcal{F}_{V}(Y)$ and multiply them to the $Y^{2 i}$ s in the table.

Although the starting relation $\mathcal{F}_{V}(Y)=Y^{D}+\sum_{0<i<j .2^{i}+2^{j}<D} a_{i j} Y^{2^{i}+2^{j}}$ is a sparse polynomial, the polynomials become dense quickly in the course of the raising process. However, the number of terms in the polynomials is restricted by $D$ because of $\bmod \mathcal{F}_{V}(Y)$. We expect the number of terms to be in average $D$ during the computation.

The number of field multiplications needed to compute the $Y^{2 i}$ table is $O(2$. $\left.D^{2}\right)$. To raise $Y^{2^{m}}$ to $Y^{2^{n}}$ we need $O((n-m) \cdot D)$ squarings and $O\left((n-m) \cdot D^{2}\right)$ multiplications. We can further reduce the number of computations needed for raising $Y^{2^{m}}$ by using a higher degree $Y^{i}$ table. For example, if we raise $Y^{2^{m}}$ to $Y^{2^{4 m}}$ in one step, we need only $O((n-m) \cdot D)$ squarings and $O\left(\frac{(n-m)}{2} \cdot D^{2}\right)$ multiplications. However, the computational effort for preparing the $Y^{i}$ table increases. Table 8 shows the time needed to compute $\operatorname{gcd}\left(Y^{2^{n}}-Y, \mathcal{F}(Y)\right)$ on two different CPUs.

\begin{tabular}{|c|c|c|c|c|}
\hline scheme & $\begin{array}{c}\text { security } \\
\text { level (bit) }\end{array}$ & $\begin{array}{c}\text { public key } \\
\text { size }(\mathrm{kB})\end{array}$ & $\begin{array}{c}\text { private key } \\
\text { size }(\mathrm{kB})\end{array}$ & $\begin{array}{c}\text { time needed for } \\
\text { inverting } \mathcal{F} \text { (kilo-cycles) }\end{array}$ \\
\hline HFEv- $(96,5,6,6)$ & 80 & 61.6 & 3.1 & $72 / 76 / 55$ \\
\hline HFEv- $(95,9,5,5)$ & 80 & 59.2 & 3.0 & $159 / 135 / 79$ \\
\hline HFEv- $(94,17,4,4)$ & 80 & 56.8 & 2.9 & $533 / 453 / 274$ \\
\hline HFEv- $(127,9,4,6)$ & 120 & 139.2 & 5.2 & $170 / 156 / 128$ \\
\hline HFEv- $(103,129,3,4)$ & 80 & 71.9 & 3.1 & $25,793 / 20,784 / 12,630$ \\
\hline
\end{tabular}

[1] AMD Opteron 6212, 2.5 GHz (Bulldozer)/ Intel Xeon CPU E5-2620, $2.0 \mathrm{GHz}$ (Sandy Bridge) / Intel Xeon E3-1245 v3, 3.4 GHz (Hashwell)

Table 8. Key sizes of HFEv- schemes and running time of $\operatorname{gcd}\left(X^{2^{n}}-X, \mathcal{F}(X)\right)$

\subsection{Experiments and Comparison}

Table 9 shows key and signature sizes as well as the running times of signature generation and verification of Gui and compares these data with those of some standard signature schemes. The data are benchmarked according to specifications given by the eBACS project [3]. 


\begin{tabular}{|c|r|r|r|r|r|r|}
\hline Scheme & $\begin{array}{r}\text { security } \\
\text { level(bits) }\end{array}$ & $\begin{array}{r}\text { public key } \\
\text { size (Bytes) }\end{array}$ & $\begin{array}{r}\text { private key } \\
\text { size (Bytes) }\end{array}$ & $\begin{array}{r}\text { signature } \\
\text { size(bits) }\end{array}$ & $\begin{array}{r}\text { signing time } \\
\text { (k-cycles) }\end{array}$ & $\begin{array}{r}\text { verification time } \\
\text { (k-cycles) }{ }^{1}\end{array}$ \\
\hline Gui-96 $(96,5,6,6)$ & 80 & 63,036 & 3,175 & 126 & $603 / 569 / 238$ & $97 / 70 / 62$ \\
\hline Gui-95 $(95,9,5,5)$ & 80 & 60,600 & 3,053 & 120 & $1,417 / 1,441 / 602$ & $91 / 60 / 58$ \\
\hline Gui-94 $(94,17,4,4)$ & 80 & 58,212 & 2,943 & 124 & $5,800 / 5,480 / 2,495$ & $118 / 74 / 71$ \\
\hline Gui-127 $(127,9,4,6)$ & 120 & 142,576 & 5,350 & 163 & $2,368 / 2,183 / 1,080$ & $220 / 121 / 122$ \\
\hline \hline QUARTZ $(103,129,3,4)$ & 80 & 73,626 & 3,174 & 128 & $302,882 / 315,716 / 128,736$ & $145 / 84 / 86$ \\
\hline \hline RSA-1024 & 80 & 128 & 128 & 128 & $2,080 / 1,058 /-2$ & $74 / 32 /-$ \\
\hline RSA-2048 & 112 & 256 & 256 & 256 & $8,834 / 5,347 /-$ & $138 / 76 /-$ \\
\hline \hline ECDSA P160 & 80 & 40 & 60 & 320 & $1,283 / 558 /-$ & $1,448 / 635 /-$ \\
\hline ECDSA P192 & 96 & 48 & 72 & 384 & $1,513 / 773 /-$ & $1,715 / 867 /-$ \\
\hline ECDSA P256 & 128 & 64 & 96 & 512 & $830 / 388 /-$ & $2,111 / 920 /-$ \\
\hline
\end{tabular}

${ }^{1}$ AMD Opteron 6212, $2.5 \mathrm{GHz}$ (Bulldozer) / Intel Xeon CPU E5-2620, $2.0 \mathrm{GHz}$ (Sandy Bridge) / Intel Xeon E3-1245 v3, 3.4 GHz (Hashwell)

${ }^{2}$ For the classical signature schemes RSA and ECDSA, we do not have data for the Hashwell processor

Table 9. Comparison between Gui and standard signature schemes

We should note that the timings for Gui given by Table 9 are for C programs with a few intrinsic function calls of PCLMULQDQ. The PKCs benchmarked in the eBACs project also do not represent optimal implementations of RSA and ECC. We present these numbers in an effort to compare apples to apples by using only reference implementations.

\section{Conclusion and Future Work}

In this paper, we analyzed the behavior of direct attacks against HFEv- based signature scheme. Experiments show that, even for low degree HFE polynomials in use, we can obtain adequate security levels by increasing the numbers $a$ and $v$ of Minus equations and Vinegar variables. Furthermore we find that the upper bound on the degree of regularity found by Ding and Yang in [11] is relatively tight. From our results we derive design principles for the construction of HFEvbased signature schemes, which lead to both secure and efficient schemes. We apply our principles in the construction of our new HFEv- based signature scheme Gui, which is more than 100 times faster than the original QUARTZ scheme. Furthermore we show that the performance of our scheme is highly comparable to that of standard signature schemes, including signatures on elliptic curves.

The most obvious future work would be to create for every common existing platform an optimal implementation of HFEv- (Gui) and compare it with some of the best optimized code for ECC and RSA, such as Ed25519 [2]. The other would be to verify such optimal Gui code for formal correctness. In short, we 
believe that there is still much work to be done about the HFEv- digital signature schemes.

\section{References}

1. D.J. Bernstein, J. Buchmann, E. Dahmen (eds.): Post Quantum Cryptography. Springer, 2009.

2. D. J. Bernstein, N. Duif, T. Lange, P. Schwabe, and B.-Y. Yang, High-speed highsecurity signatures, J. Cryptographic Engineering 2:2, pp. 77-89 (2012).

3. D.J. Bernstein and T. Lange (editors). eBACS: ECRYPT Benchmarking of Cryptographic Systems. http://bench.cr.yp.to, accessed 14 May 2014.

4. A. Bogdanov, T. Eisenbarth, A. Rupp, C. Wolf. Time-area optimized public-key engines: MQ-cryptosystems as replacement for elliptic curves? CHES 2008, LNCS vol. 5154, pp. 45-61. Springer, 2008.

5. A.I.T. Chen, M.-S. Chen, T.-R. Chen, C.-M. Cheng, J. Ding, E. L.-H. Kuo, F. Y.-S. Lee, B.-Y. Yang. SSE implementation of multivariate PKCs on modern x86 cpus. CHES 2009, LNCS vol. 5747, pp. 33 - 48. Springer, 2009.

6. N.T. Courtois, M. Daum, P. Felke: On the Security of HFE, HFEv- and QUARTZ. PKC 2003, LNCS vol. 2567, pp. 337 - 350. Springer 2003.

7. J. Ding, J. E. Gower, D. S. Schmidt: Multivariate Public Key Cryptosystems. Springer, 2006.

8. J. Ding, T. Hodges: Inverting HFE Systems Is Quasi-Polynomial for All Fields. CRYPTO 2011, LNCS vol. 6841, pp. 724-742. Springer 2011.

9. J. Ding, T. Kleinjung: Degree of regularity for HFE-. IACR eprint 2011/570.

10. J. Ding, D. S. Schmidt: Rainbow, a new multivariate polynomial signature scheme. ACNS 2005, LNCS vol. 3531, pp. 164-175. Springer 2005.

11. J. Ding, B.Y. Yang: Degree of Regularity for HFEv and HFEv-. PQCrypto 2013, LNCS vol. 7932, pp. 52-66. Springer, 2013.

12. J.C. Faugère: A new efficient algorithm for computing Gröbner bases (F4). Journal of Pure and Applied Algebra 139, pp. 61-88 (1999).

13. A. Fog: Instruction tables: Lists of instruction latencies, throughputs and micro-operation breakdowns for Intel, AMD and VIA CPUs. http://www.agner.org/optimize/, 7th Dec 2014.

14. M. R. Garey and D. S. Johnson: Computers and Intractability: A Guide to the Theory of NP-Completeness. W.H. Freeman and Company 1979.

15. Intel Corporation, Hashwell Cryptographic Performance. http://www.intel.com/content/dam/www/public/ us/en/documents/white-papers/haswell-cryptographic-performance-paper.pdf

16. D. Kravitz: Digital Signature Algorithm. US patent 5231668 (July 1991).

17. A. Kipnis, L. Patarin, L. Goubin: Unbalanced Oil and Vinegar Schemes. EUROCRYPT 1999, LNCS vol. 1592, pp. 206-222. Springer 1999.

18. A. Kipnis, A. Shamir: Cryptanalysis of the HFE Public Key Cryptosystem. CRYPTO 99, LNCS vol. 1666, pp. 19 - 30. Springer 1999.

19. M. S. E. Mohamed, J. Ding and J. Buchmann: Towards Algebraic Cryptanalysis of HFE Challenge 2, ISA 2011, Communications in Computer and Information Science vol. 200, pp. 123-131. Springer 2011.

20. T. Matsumoto, H. Imai: Public quadratic polynomial-tuples for efficient signatureverification and message-encryption. EUROCRYPT 1988. LNCS vol. 330, pp. 419453. Springer 1988. 
21. J. Patarin, N. Courtois, L. Goubin: QUARTZ, 128-Bit Long Digital Signatures. CTRSA 2001, LNCS vol. 2020, pp. 282-297. Springer, 2001.

22. J. Patarin, N. Courtois, L. Goubin: Flash, a fast multivariate signature algorithm. CTRSA 2001, LNCS vol. 2020, pp. 298 - 307. Springer, 2001.

23. J. Patarin: Cryptanalysis of the Matsumoto and Imai public key scheme of Eurocrypt 88. CRYPTO 95. LNCS vol. 963, pp. 248 - 261. Springer 1995.

24. C. Richards: Algorithms for Factoring Square-Free Polynomials over Finite Fields. Master Thesis, Simon Fraser University (Canada), 2009.

25. R. L. Rivest, A. Shamir, L. Adleman: A Method for Obtaining Digital Signatures and Public-Key Cryptosystems. Commun. ACM 21 (2), pp. 120-126 (1978).

26. P. Shor: Polynomial-Time Algorithms for Prime Factorization and Discrete Logarithms on a Quantum Computer, SIAM J. Comput. 26 (5), pp. 1484 - 1509 (1997).

27. J. Taverne, A. Faz-Hernàndez, D. F. Aranha, F. Rodrquez-Henrïquez, D. Hankerson, J. López: Software Implementation of Binary Elliptic Curves: Impact of the Carry-Less Multiplier on Scalar Multiplication. CHES 2011, LNCS vol. 6917, pp. 108-123. Springer 2011.

28. http://en.wikipedia.org/wiki/ File: CMOC_Treasures_of_Ancient_China_exhibit_white_pottery_gui_1.jpg. 